\title{
EATRHQUAKE RESPONSE OF REINFORCED COCRETE MULTI STOREY BUILDING WITH BASE ISOLATION
}

\author{
Swathirani.K.S ${ }^{1}$, Muralidhara.G.B ${ }^{2}$, Santoshkumar.N.B ${ }^{3}$ \\ ${ }^{1}$ Assistant Professor, Civil Engineering Department, East West Institute of Technology, Karnataka, India \\ ${ }^{2}$ Lecturer, Civil Engineering Department, University of Gondar, Ethiopia \\ ${ }^{3}$ Design Engineer, Sobha Developers, Karnataka, India
}

\begin{abstract}
A seismic base isolation is an earthquake resistant design method that is based on reducing the seismic demand and also the ductility demand. The basic concept of base isolation system is to increase the natural time period of the fixed base building and to protect the structure from the seismic effect. The main purpose of this work is to study the dynamic response of structure when subjected to different isolators. For this purpose, a three dimensional response spectrum analysis and time history analysis is performed using a commercial structural analysis software package called as SAP 2000 to study the influence of isolation damping on building. The main objective is to make a comparison between the fixed base building and various isolation systems such as friction pendulum isolator, lead rubber bearing (LRB) isolator and high damping rubber isolator, subjected to strong earthquakes to achieve an optimal design of the base isolation system. SAP 2000, a popular large scale explicit finite element analysis software used to stimulate the behavior of base isolated structure. In the present work, it was found that base isolation substantially increases the time period of structure and hence correspondingly reduces the base shear. The top displacement for fixed and isolated cases conspicuously shows the reduction in the case of isolated frame. The study shows that the bending moment and shear force values are reduced for base isolated frame when compared to fixed base building. From design consideration the steel requirement is reduced in base isolated structures when compared to fixed base building.
\end{abstract}

Key Words: Isolators, SAP2000

\section{INTRODUCTION}

The previous Earthquake data provides enough evidence for behavior of different types of structures under different seismic conditions and foundation aspects has become stuff for Engineers and Scientists. This has given various types of innovative techniques to save structures from seismic effects. Among those, Base Isolation is one of the recent technique. The main aim of base isolation is to provide flexibility and dissipation of energy by incorporating the isolated devices so called isolators, which is provided between the foundation and the super structure. Thus, base isolation essentially dissociates the building from the ground during seismic excitation. The use of flexible layer by base isolation systems at the base of the structure will allow relative displacements between the foundation and the superstructure. Addition of isolation layer elongates the fundamental time period of the structure so as to move away from the ascendant time periods of motion of ground, it means that decreasing the acceleration induced in the structure.

\subsection{Basic Concept of Base Isolation}

The basic concept of base isolation is to protect the structure from the damage caused due to seismic forces by establishing a support isolating the building from the ground movement. In simple way, the structure is isolated from its foundations. Due to seismic forces, there will be movement in ground and this ground movement tends to damage the structure. So, the concept is literal to isolate the structure from the ground. So that we can control the movement of building. By using the Base isolation systems, such as sliding and rubber isolator bearing systems, reduces the response of structure, but there will be increase in base displacements in near-fault motions.

To limit the bearing displacement nonlinear passive dampers are provided; however, this enhances the forces in the superstructure. The base isolation concept is explained by an example of building resting on frictionless roller. When the ground moves, the rollers freely roll, but the superstructure will not move. Because of this no force is transmitted to the superstructure due to the movement of the ground; literally, the superstructure does not experience the seismic forces. Now, if the same structure is rested on adjustable pads which is having resistance against lateral movements, then some effect of the ground movement will be transferred to the above building. If the adjustable pads are properly chosen, the forces induced by the ground movement will be a few times lesser than that experienced by the structure built directly on ground, namely a fixed base building. The adjustable pads are called base-isolators.

Therefore, the structures secured by means of these devices are called base-isolated buildings. The important feature of this technic is that it introduces flexibility in the structure. As a result, a medium-rise masonry building or reinforced concrete building becomes extremely flexible. These isolators are also designed to absorb energy by that damping is added to the system. This helps in further decrease the 
earthquake response of the building. Many commercial brands of base isolators are widely available, and many of them look like large rubber pads, although there are other types that are based on sliding of one part of the building relative to the other. A peer study is required to select the most suitable type of device for a specific building. Also, base isolation is not acceptable for all buildings. Base isolation are more acceptable for low to medium-rise buildings rested on hard soil. But not for High-rise buildings or buildings rested on soft soil.

\subsection{Types Of Isolation Components (Isolators)}

\section{a) Lead-Rubber Bearings}

A lead-rubber bearing is one of the type of elastomeric bearing. The lead core provides rigidity under service loads and energy dissipation under high lateral loads. The entire bearing is encased in cover rubber to provide environmental protection. When subjected to low lateral loads, the lead rubber bearing is stiff both laterally and vertically. The lateral stiffness results from the high elastic stiffness of the lead plug and the vertical rigidity results from the steelrubber construction of the bearing

\section{b) High-Damping Natural Rubber Bearings}

The high damping rubber bearing is one of the type of elastomeric bearings where the elastomer used (either natural or synthetic rubber) provides a significant amount of damping. This type of bearing consists of thin layers of high damping rubber and steel plates built in alternative layers. Here rubber reinforced with steel plates provides stable support for structures. Using this type of system seismic vibrations is converted to low speed motion. The outcome of this system is after earthquake the building will regain its original position.

\section{c) Friction Pendulum System}

Friction pendulum (FP) isolators is one of the special type of sliding isolator, which executes the same principle of simple pendulum. The isolator setup consists of a concave sliding surface and an articulated slider that is coated with a low friction composite material. When the concave spherical bearings are placed at a particular support point, there will be a sway in a structure same like a gentle pendulum motion at the time of shaking of ground. This system is featured as substantial under severe environmental conditions and insensitivity to the frequency content of ground motion.

\section{NUMERICAL EXAMPLE}

\subsection{Structural Models Considered}

In this analytical study, three-dimensional RC building considered, incorporated with different base isolation devices under each column which have been investigated. Building considered is as shown in Fig 1, is an actual eightstorey RC building of $21 \mathrm{~m} \times 26.4 \mathrm{~m}$ in plan ( $\mathrm{x}$ and $\mathrm{y}$ axes) dimensions and $29.7 \mathrm{~m}$ in height ( $\mathrm{z}$ axis). This building is connected with a total number of 50 base isolation devices under each column. For the analysis, structural analysis software SAP version 14.2.0 is used. By using this software, each floor slab is modeled as a rigid diaphragm, so that each floor is represented by three degrees of freedom: two lateral degrees of freedom in the $\mathrm{x}$ and $\mathrm{y}$ directions and a rotational degree of freedom about the vertical axis. These degrees of freedoms are located at the center of the floor mass, which is the same at every floor. Here the structural models used are having symmetric floor plans. The analysis is carried out using response spectrum and time history analysis. According to variations in the design parametric characteristics the relative effectiveness of the isolation systems is investigated.

\subsection{Dimensions of 3d Frame}

Isometric view \& Top view of reinforced cement concrete, C-shaped 8 storey frame is as shown in the Fig.1 \& Fig.2 respectively.

Storey height $=3.4 \mathrm{~m}$ is being modeled in SAP-2000.

Sizes of the structural members are as follows:

Beam size1: $230 \mathrm{~mm} \times 350 \mathrm{~mm}$.

Beam size2: $230 \mathrm{~mm}$ x $400 \mathrm{~mm}$.

Column size1: $230 \mathrm{~mm}$ x $900 \mathrm{~mm}$.

Column size2: $230 \mathrm{~mm} \times 1000 \mathrm{~mm}$.

Roof slab: $\quad 125 \mathrm{~mm}$ thick.

The beam \& column sizes are kept same for both fixed and isolated frame.

Isometric view \& Top view of reinforced cement concrete, $\mathrm{C}$-shaped 8 storey frame are as shown in the figures.

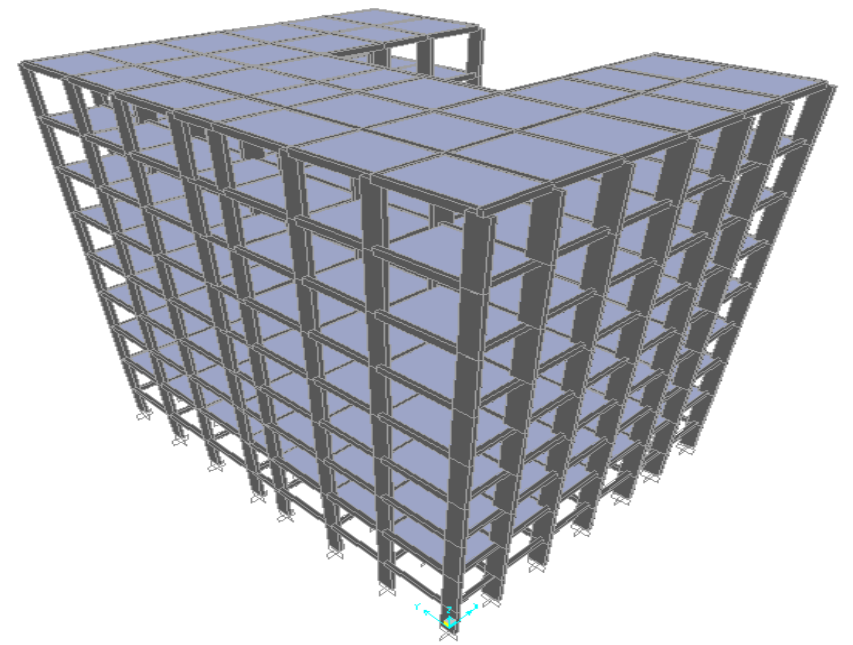

Fig -1: Isometric view of C-shaped frame

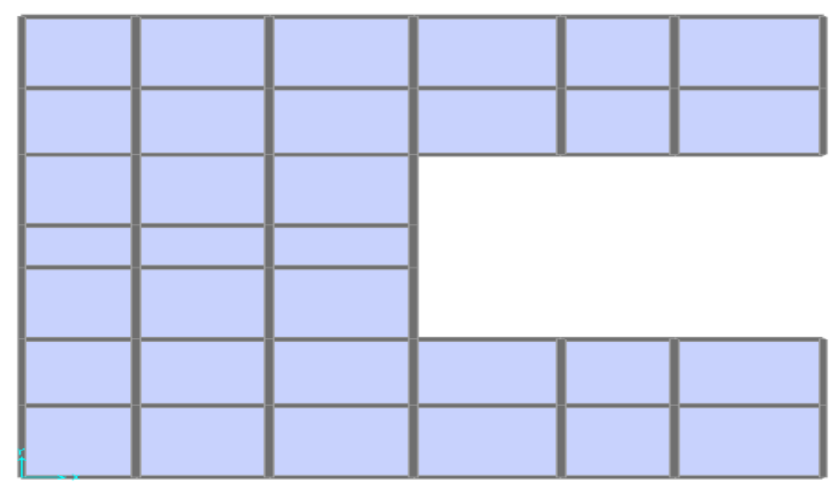

Fig -2: Top view of the C-shaped frame 
The modelling of base isolators has been done in SAP using Joint 2 link element type are,

Type I: Friction pendulum isolator frame with all isolators of uniform stiffness.

Type II: Rubber Isolated frame with all isolators of uniform stiffness.

Type III: Friction isolator frame with outer columns of one stiffness and inner columns of another stiffness.

Type IV: High damping rubber Isolated frame with all isolators of uniform stiffness.

The seismic isolators in the system are defined as NL link components $0.5 \mathrm{~m}$ in length placed between the fixed base and the columns. The parameters selected to define the utilized isolators in the SAP2000 program are as follows:

Table -1: Isolator properties

\begin{tabular}{|l|l|l|l|l|}
\hline & $\begin{array}{l}\text { Type } \\
\text { I }\end{array}$ & $\begin{array}{l}\text { Type } \\
\text { II }\end{array}$ & $\begin{array}{l}\text { Type } \\
\text { III }\end{array}$ & $\begin{array}{l}\text { Type } \\
\text { IV }\end{array}$ \\
\hline $\mathrm{K}_{1}$ & 15000000 & 1500000 & 15000000,5000000 & 350.4 \\
\hline $\mathrm{K}_{2}$ & 15000 & 2500 & 15000,2000000 & 9.32 \\
\hline $\mathrm{K}_{3}$ & 15000 & 2500 & 15000,2000000 & 9.32 \\
\hline $\mathrm{KE}_{2}$ & 750 & 800 & 750,150 & 0.83 \\
\hline $\mathrm{KE}_{3}$ & 750 & 800 & 750,150 & 0.83 \\
\hline $\mathrm{FY}_{2}$ & 0.03 & 80 & $0.03,0.04$ & - \\
\hline $\mathrm{FY}_{3}$ & 0.03 & 80 & $0.03,0.04$ & - \\
\hline $\mathrm{RK}_{2}$ & 0.05 & 0.1 & $0.05,0.1$ & - \\
\hline $\mathrm{RK}_{3}$ & 0.05 & 0.1 & $0.05,0.1$ & - \\
\hline $\mathrm{A}_{2}$ & 40 & - & 40,900 & - \\
\hline $\mathrm{A}_{3}$ & 40 & - & 40,900 & - \\
\hline $\mathrm{R}_{2}$ & 2.23 & & $2.23,0.0$ & \\
\hline $\mathrm{R}_{3}$ & 2.23 & & $2.23,0.0$ & \\
\hline $\mathrm{Wh}$ & & & & \\
\hline
\end{tabular}

Where,

$\mathrm{K}_{1} \quad$ spring stiffness along axis 1(Axial)

$\mathrm{K}_{2} \quad$ Initial Spring stiffness along axis 2

$\mathrm{K}_{3} \quad$ Initial Spring stiffness along axis 3

$\mathrm{KE}_{2} \quad$ Spring Effective stiffness along axis 2

$\mathrm{KE}_{3} \quad$ Spring Effective stiffness along axis 3

$\mathrm{FY}_{2} \quad$ Yield Force along axis 2

$\mathrm{FY}_{3} \quad$ Yield Force along axis 3

$\mathrm{RK}_{2} \quad$ Post-Yield stiffness ratio along axis 2

$\mathrm{RK}_{3} \quad$ Post-Yield stiffness ratio along axis 3

$\mathrm{A}_{2} \quad$ Coefficient controlling friction axis 2

$\mathrm{A}_{3} \quad$ Coefficient controlling friction axis 3

$\mathrm{R}_{2} \quad$ Radius of Contact 2 direction

$\mathrm{R}_{3} \quad$ Radius of Contact 3 direction

The joint local 1- 2-3 coordinate system is normally the same as the global X- Y-Z coordinate system.

\subsection{Seismic Loading:}

For response spectrum analysis, the response spectrum given in IS 1893-2002

Zone $\mathrm{V}, \mathrm{Z}=0.36$
Importance factor $\mathrm{I}=1.0$

Response reduction factor $\mathrm{R}=5$.

Soil type: Type II (medium)

Time period $\mathrm{T}=\frac{0.09 * h}{\sqrt{D}}$

$$
=\frac{0.09 * 29.7}{\sqrt{21}}=0.5833 \mathrm{sec} \text {. }
$$

Table -2: Total base shear values (KN)

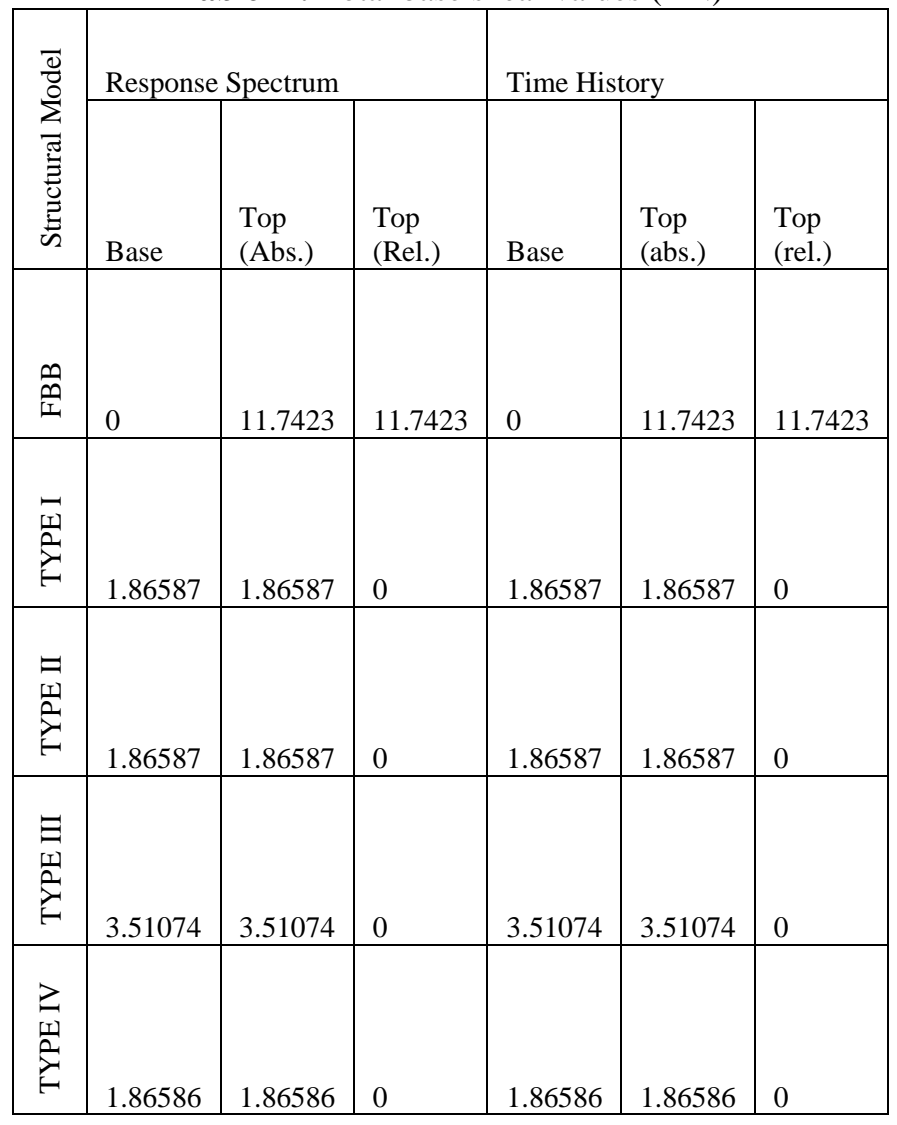

Damping in analysis for both fixed base \& base isolated structure is taken as $5 \%$ as the default value in IS 18932002 response spectrum. Linear isolator is being used so no additional damping will be there due to the damping of isolator.

\section{RESULTS OF ANALYSIS CARRIED OUT:}

General:

The four structural models under consideration are analyzed in SAP 2000 (Ver. 14.2) for Response Spectrum (IS 18932002) \& Time History (EL-Centro, NS-Component 1940) and analysis results are given in following tables (Table -2 to Table -20).

Table -3: Fundamental time period in sec.

\begin{tabular}{|l|l|l|l|l|}
\hline \multirow{2}{*}{$\begin{array}{l}\text { Structural } \\
\text { Model }\end{array}$} & \multicolumn{2}{|l|}{ Response Spectrum } & \multicolumn{2}{l|}{ Time History } \\
\cline { 2 - 5 } & $\mathrm{X}$ & $\mathrm{Y}$ & $\mathrm{X}$ & $\mathrm{Y}$ \\
\hline FBB & 5978.962 & 6410.898 & 5978.962 & 6410.898 \\
\hline
\end{tabular}




\begin{tabular}{|l|l|l|l|l|}
\hline TYPE I & 1191.809 & 1405.681 & 560.344 & 672.842 \\
\hline TYPE II & 886.001 & 1048.611 & 384.337 & 409.453 \\
\hline TYPE III & 501.617 & 596.730 & 143.099 & 120.089 \\
\hline TYPE IV & 731.684 & 866.277 & 265.665 & 269.142 \\
\hline
\end{tabular}

The total base shear is given in Table -2, for both response spectrum \& Time History analysis.

Table -4 Base and top displacements (in $\mathrm{mm}$ ):

\begin{tabular}{|l|l|l|}
\hline $\begin{array}{l}\text { Structural } \\
\text { Model }\end{array}$ & Response Spectrum & Time History \\
\hline FBB & 0.29259 & 0.29267 \\
\hline TYPE I & 2.76796 & 2.76918 \\
\hline TYPE II & 2.68007 & 2.68007 \\
\hline TYPE III & 3.30933 & 3.30933 \\
\hline TYPE IV & 2.63119 & 2.63019 \\
\hline
\end{tabular}

Compares the fundamental time periods which is obtained from modal analysis, for the 4 cases under consideration. Compares the base \& top displacements, obtained from SAP for both response spectrum \& Time History analysis. For each case the nodal displacement value which gives maximum top relative and absolute displacement are compared.

Table -5: Bending Moment in Columns (KN-m):

\begin{tabular}{|l|l|l|l|l|l|}
\hline \multicolumn{5}{|c|}{ Column Bending Moment Values in Kn-m } \\
\hline $\begin{array}{l}\text { COL } \\
\text { NO }\end{array}$ & FBB & $\begin{array}{l}\text { TYPE } \\
\text { I }\end{array}$ & $\begin{array}{l}\text { TYPE } \\
\text { II }\end{array}$ & $\begin{array}{l}\text { TYPE } \\
\text { III }\end{array}$ & $\begin{array}{l}\text { TYPE } \\
\text { IV }\end{array}$ \\
\hline C1 & 542.213 & 2.796 & 2.827 & 2.819 & 2.902 \\
\hline C2 & 578.173 & 2.829 & 2.837 & 2.85 & 2.855 \\
\hline C3 & 696.828 & 3.714 & 3.662 & 3.668 & 3.527 \\
\hline C4 & 490.705 & 2.998 & 2.979 & 3.013 & 2.935 \\
\hline C5 & 424.125 & 2.861 & 2.9 & 2.896 & 3.003 \\
\hline C6 & 541.717 & 2.697 & 2.782 & 2.705 & 2.892 \\
\hline C7 & 695.333 & 3.603 & 3.585 & 3.585 & 3.533 \\
\hline C8 & 706.416 & 3.81 & 3.767 & 3.78 & 3.65 \\
\hline C9 & 712.818 & 4.004 & 3.968 & 3.974 & 3.856 \\
\hline C10 & 714.492 & 3.759 & 3.713 & 3.71 & 3.59 \\
\hline
\end{tabular}

The bending moment values for ground storey columns for response spectrum analysis are given in above Table -5 .

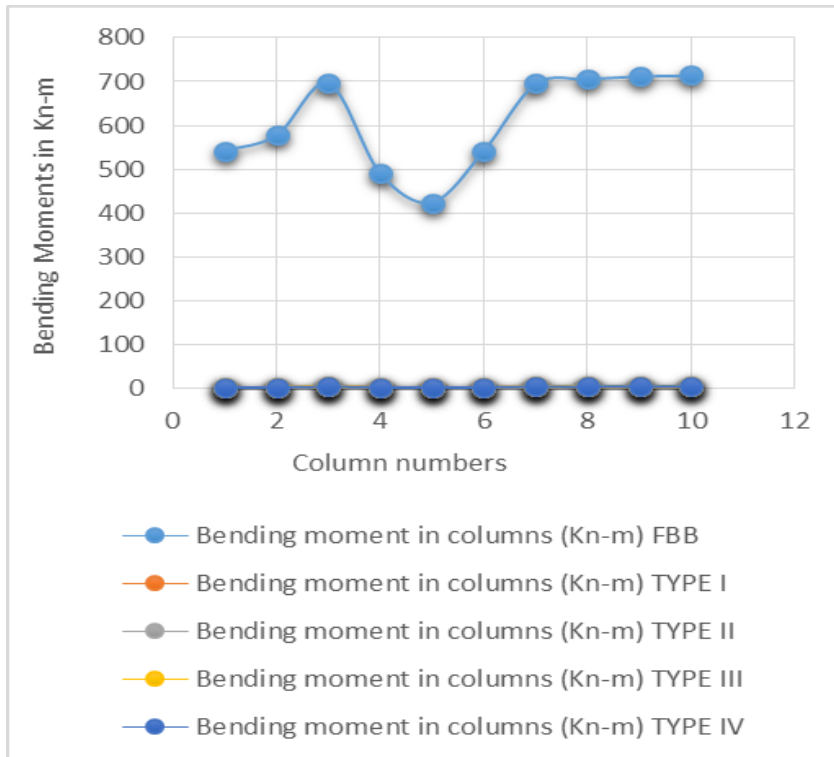

Chart -1: Bending Moment Values of Ground Storey Column

The above chart shows the bending moment values for ground storey columns. From the chart we can see that the bending moment values for base isolated buildings is much lesser than that of the fixed base building. The bending moment values for base isolated buildings are almost having same values.

Table -6: Column B.M Values (KN-m) w.r.t FBB: Column Bending Moments (KN-m) Values w.r.t Fixed Base Building

\begin{tabular}{|l|l|l|l|l|}
\hline $\begin{array}{l}\text { COL } \\
\text { NO }\end{array}$ & TYPE I & TYPE II & TYPE III & TYPE IV \\
\hline C1 & -539.417 & -539.386 & -539.394 & -539.311 \\
\hline C2 & -575.344 & -575.336 & -575.323 & -575.318 \\
\hline C3 & -693.114 & -693.166 & -693.16 & -693.301 \\
\hline C4 & -487.707 & -487.726 & -487.692 & -487.77 \\
\hline C5 & -421.264 & -421.225 & -421.229 & -421.122 \\
\hline C6 & -539.02 & -538.935 & -539.012 & -538.825 \\
\hline C7 & -691.73 & -691.748 & -691.748 & -691.8 \\
\hline C8 & -702.606 & -702.649 & -702.636 & -702.766 \\
\hline C9 & -708.814 & -708.85 & -708.844 & -708.962 \\
\hline C10 & -710.733 & -710.779 & -710.782 & -710.902 \\
\hline
\end{tabular}

- - ve indicates that bending moment decreases.

- + ve indicates that bending moment Increases 
Table -7: Column shear Values (KN):

\begin{tabular}{|l|l|l|l|l|l|}
\hline \multicolumn{5}{|l|}{ Column Shear Values In KN } & \\
\hline $\begin{array}{l}\text { COL } \\
\text { NO }\end{array}$ & FBB & TYPE I & TYPE II & TYPE III & TYPE IV \\
\hline C1 & 339.373 & 7.085 & 8.014 & 7.949 & 10.425 \\
\hline C2 & 361.659 & 6.992 & 7.943 & 7.866 & 10.376 \\
\hline C3 & 436.003 & 1.689 & 3.253 & 2.668 & 7.977 \\
\hline C4 & 312.612 & 5.164 & 5.8 & 5.64 & 7.704 \\
\hline C5 & 269.708 & 3.334 & 4.435 & 4.13 & 7.53 \\
\hline C6 & 338.7 & 4.967 & 7.281 & 4.564 & 9.978 \\
\hline C7 & 433.895 & 3.161 & 3.455 & 3.226 & 4.371 \\
\hline C8 & 441.803 & 1.684 & 3.274 & 2.687 & 8.013 \\
\hline C9 & 444.997 & 1.023 & 1.369 & 1.103 & 2.741 \\
\hline C10 & 448.054 & 2.173 & 2.459 & 2.238 & 3.812 \\
\hline
\end{tabular}

Base shear values in columns for response spectrum analysis are given in the above Table -7 .

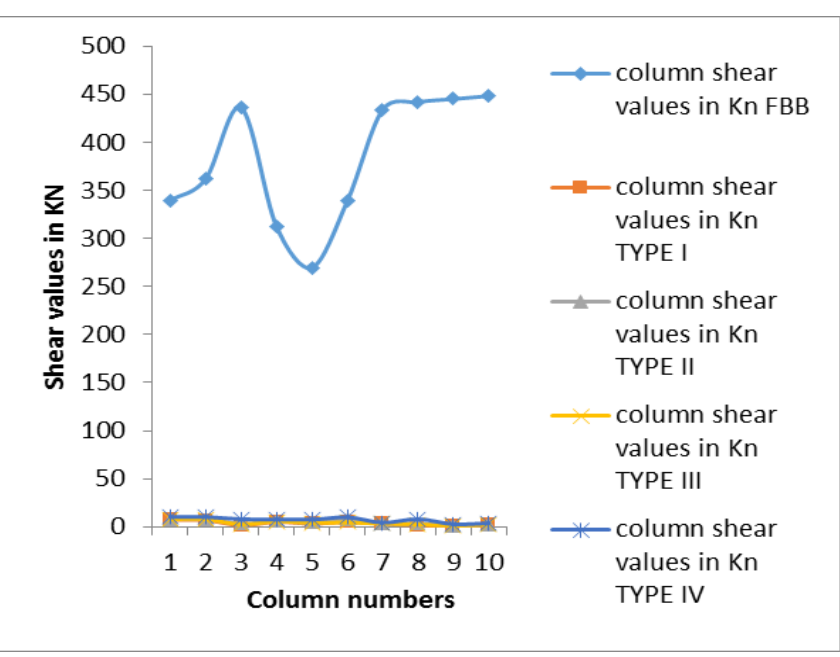

Chart -2: Column shear values of ground storey

Table -8: Column shear Values $(\mathrm{KN})$ :

Column Shear Values (KN) w.r.t Fixed Base Building

\begin{tabular}{|l|l|l|l|l|}
\hline $\begin{array}{l}\text { COL } \\
\text { NO }\end{array}$ & TYPE I & TYPE II & TYPE III & TYPE IV \\
\hline C1 & -332.288 & -331.359 & -331.424 & -328.948 \\
\hline C2 & -354.667 & -353.716 & -353.793 & -351.283 \\
\hline C3 & -434.314 & -432.75 & -433.335 & -428.026 \\
\hline C4 & -307.448 & -306.812 & -306.972 & -304.908 \\
\hline C5 & -266.374 & -265.273 & -265.578 & -262.178 \\
\hline C6 & -333.733 & -331.419 & -334.136 & -328.722 \\
\hline C7 & -430.734 & -430.44 & -430.669 & -429.524 \\
\hline C8 & -440.119 & -438.529 & -439.116 & -433.79 \\
\hline C9 & -443.974 & -443.628 & -443.894 & -442.256 \\
\hline C10 & -445.881 & -445.595 & -445.816 & -444.242 \\
\hline
\end{tabular}

Table -9: Rebar Percentage of Steel in Column

\begin{tabular}{|l|l|l|l|l|l|}
\hline \multicolumn{6}{|l|}{ Rebar Percentage (\%) Of Steel In Ground Storey Columns } \\
\hline $\begin{array}{l}\text { COL } \\
\text { NO }\end{array}$ & FBB & TYPE I & TYPE II & TYPE III & TYPE IV \\
\hline C1 & 2.3 & 0.83 & 0.87 & 0.86 & 1.01 \\
\hline C2 & 2.5 & 0.88 & 0.89 & 0.91 & 0.91 \\
\hline C3 & 3.54 & 2.42 & 2.33 & 2.34 & 2.09 \\
\hline C4 & 2.87 & 1.2 & 1.17 & 1.23 & 1.08 \\
\hline C5 & 2.4 & 0.94 & 1.02 & 1.01 & 1.21 \\
\hline C6 & 2.27 & 0.8 & 0.81 & 0.8 & 0.99 \\
\hline C7 & 3.45 & 2.23 & 2.19 & 2.19 & 2.1 \\
\hline C8 & 3.65 & 2.59 & 2.51 & 2.54 & 2.31 \\
\hline C9 & 3.8 & 2.89 & 2.83 & 2.84 & 2.66 \\
\hline C10 & 3.65 & 2.5 & 2.42 & 2.42 & 2.2 \\
\hline
\end{tabular}

Table -10: Rebar Percentage of Steel in Column Rebar Percentage (\%) Of Steel In Columns w.r.t FBB

\begin{tabular}{|l|l|l|l|l|}
\hline COL NO & TYPE I & TYPE II & TYPE III & TYPE IV \\
\hline C1 & -1.47 & -1.43 & -1.44 & -1.29 \\
\hline C2 & -1.62 & -1.61 & -1.59 & -1.59 \\
\hline C3 & -1.12 & -1.21 & -1.2 & -1.45 \\
\hline C4 & -1.67 & -1.7 & -1.64 & -1.79 \\
\hline C5 & -1.46 & -1.38 & -1.39 & -1.19 \\
\hline C6 & -1.47 & -1.46 & -1.47 & -1.28 \\
\hline C7 & -1.22 & -1.26 & -1.26 & -1.35 \\
\hline C8 & -1.06 & -1.14 & -1.11 & -1.34 \\
\hline C9 & -0.91 & -0.97 & -0.96 & -1.14 \\
\hline C10 & -1.15 & -1.23 & -1.23 & -1.45 \\
\hline
\end{tabular}

The above Table -9 gives rebar percentage of steel in column for ground storey columns w.r.t Fixed base building.

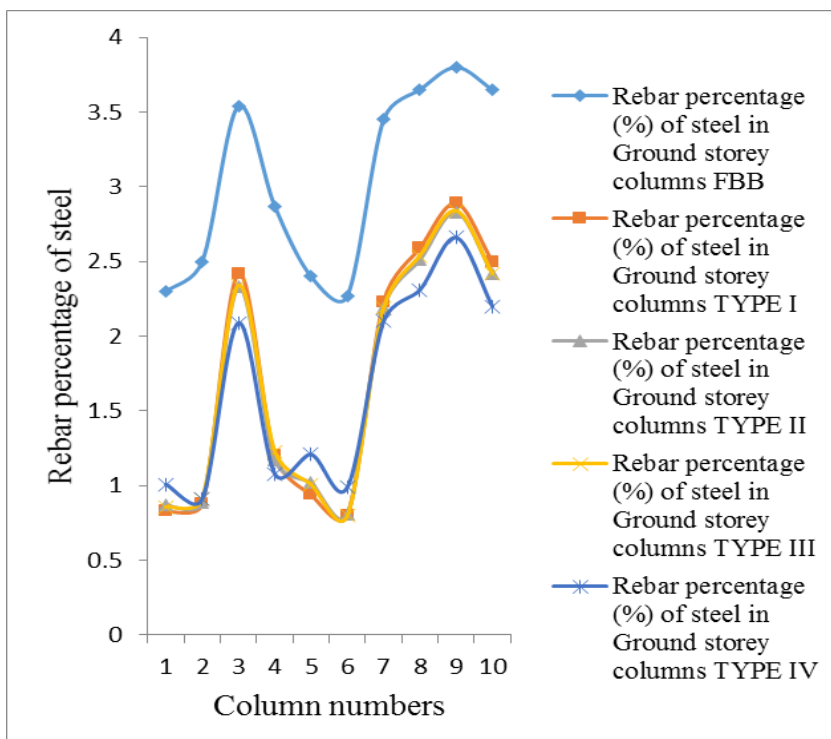

Chart -3: Rebar Percentage of Steel in Columns. 
The above Chart shows the rebar percentage of steel for ground storey columns. From the Chart we can see that the rebar percentage of steel for base isolated buildings is much lesser than that of the fixed base building. The rebar percentage of steel for TYPE I isolator is more, when compared to other isolators.

Table -11: Column Steel in $\mathrm{Kg}$

Column Longitudinal Steel In $\mathrm{Kg}$

\begin{tabular}{|l|l|l|l|l|l|}
\hline $\begin{array}{l}\text { COL } \\
\text { NO }\end{array}$ & FBB & TYPE I & TYPE II & TYPE III & TYPE IV \\
\hline C1 & 99.77 & 30 & 31.44 & 31.08 & 36.51 \\
\hline C2 & 108.44 & 31.81 & 32.17 & 32.89 & 32.89 \\
\hline C3 & 153.56 & 87.48 & 84.22 & 84.58 & 75.55 \\
\hline C4 & 112.05 & 39.04 & 38.06 & 40.01 & 35.14 \\
\hline C5 & 93.70 & 30.58 & 33.17 & 32.86 & 39.37 \\
\hline C6 & 98.47 & 28.91 & 29.28 & 28.91 & 35.78 \\
\hline C7 & 149.65 & 80.61 & 79.16 & 79.16 & 75.91 \\
\hline C8 & 158.33 & 93.62 & 90.73 & 91.81 & 83.5 \\
\hline C9 & 164.83 & 104.47 & 102.3 & 102.66 & 96.15 \\
\hline C10 & 158.33 & 90.37 & 87.48 & 87.48 & 79.52 \\
\hline
\end{tabular}

The column steel for ground storey columns are compared for all 4 cases in the above Table -11

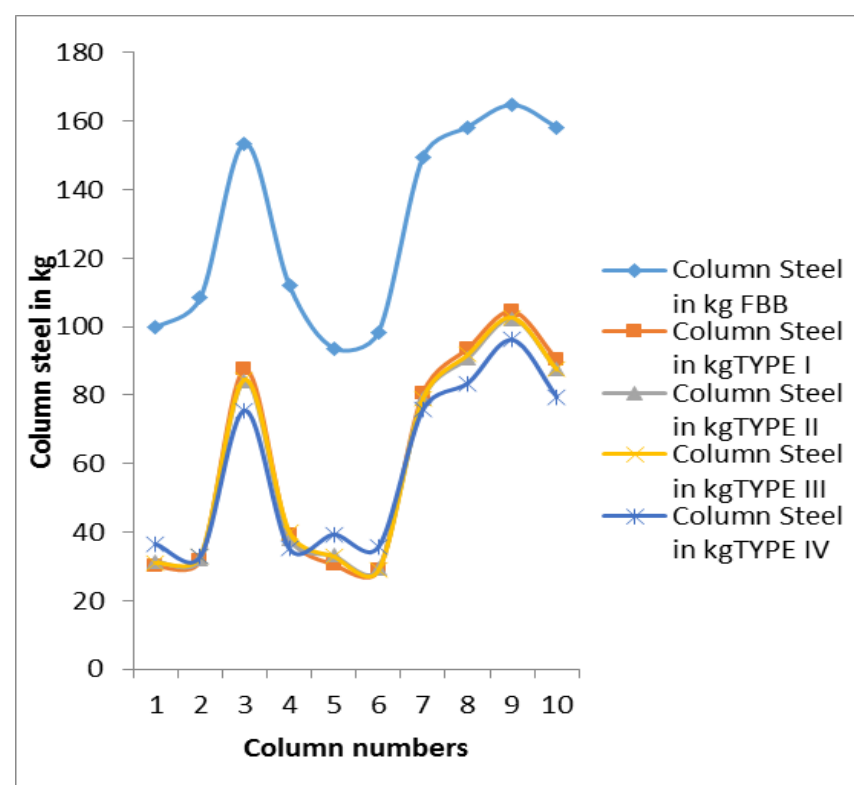

Chart -4: Column Steel Values

The above chart shows the steel values $(\mathrm{Kg})$ for ground storey columns. From the above chart, we can see that the steel values for base isolated buildings is much lesser than that of the fixed base building. The steel values for TYPE I isolator is more, when compared to other isolators.
Table -12: Column Steel in Kg w.r.t FBB

\begin{tabular}{|l|l|l|l|l|}
\hline \multicolumn{5}{|c|}{ Column Longitudinal Steel In Kg w.r.t FBB } \\
\hline $\begin{array}{l}\text { COL } \\
\text { NO }\end{array}$ & TYPE I & TYPE II & TYPE III & TYPE IV \\
\hline C1 & -69.77 & -68.33 & -68.69 & -63.26 \\
\hline C2 & -76.63 & -76.27 & -75.55 & -75.55 \\
\hline C3 & -66.08 & -69.34 & -68.98 & -78.01 \\
\hline C4 & -73.01 & -73.99 & -72.04 & -76.91 \\
\hline C5 & -63.12 & -60.53 & -60.84 & -54.33 \\
\hline C6 & -69.56 & -69.19 & -69.56 & -62.69 \\
\hline C7 & -69.04 & -70.49 & -70.49 & -73.74 \\
\hline C8 & -64.71 & -67.6 & -66.52 & -74.83 \\
\hline C9 & -60.36 & -62.53 & -62.17 & -68.68 \\
\hline C10 & -67.96 & -70.85 & -70.85 & -78.81 \\
\hline
\end{tabular}

The column steel for ground storey columns w.r.t fixed base building is given in Table -12

Table -13: Column Steel In $\mathrm{Kg} / \mathrm{m}^{3}$

\begin{tabular}{|l|l|l|l|l|l|}
\hline \multicolumn{7}{|c|}{ Column Longitudinal Steel In Kg /M } & \\
\hline $\begin{array}{l}\text { COL } \\
\text { NO }\end{array}$ & FBB & TYPE I & TYPE II & TYPE III & TYPE IV \\
\hline C1 & 180.745 & 65.225 & 68.369 & 67.583 & 79.371 \\
\hline & & & & & \\
C2 & 196.462 & 69.155 & 69.941 & 71.512 & 71.512 \\
\hline & & & & & \\
C3 & 278.19 & 190.175 & 183.103 & 183.889 & 164.242 \\
\hline C4 & 225.542 & 94.302 & 91.948 & 96.656 & 84.887 \\
\hline C5 & 188.604 & 73.877 & 80.141 & 79.382 & 95.099 \\
\hline C6 & 178.388 & 62.868 & 63.654 & 62.868 & 77.799 \\
\hline C7 & 271.118 & 175.244 & 172.101 & 172.101 & 165.028 \\
\hline C8 & 286.835 & 203.535 & 197.248 & 199.606 & 181.531 \\
\hline C9 & 298.622 & 227.11 & 222.395 & 223.181 & 209.036 \\
\hline C10 & 286.835 & 196.462 & 190.175 & 190.175 & 172.887 \\
\hline
\end{tabular}

The column steel for ground storey columns are compared for all 4 cases in the above Table -13 
Table -14: Column Steel In $\mathrm{Kg} / \mathrm{m}^{3}$ w.r.t FBB

\begin{tabular}{|l|l|l|l|l|}
\hline \multicolumn{5}{|c|}{ Column Longitudinal Steel In $\mathrm{Kg} / \mathrm{M}^{3}$ w.r.t fixed base building } \\
\hline $\begin{array}{l}\text { COL } \\
\text { NO }\end{array}$ & TYPE I & TYPE II & TYPE III & TYPE IV \\
\hline C1 & -115.52 & -112.376 & -113.162 & -101.374 \\
\hline C2 & -127.307 & -126.521 & -124.95 & -124.95 \\
\hline C3 & -88.015 & -95.087 & -94.301 & -113.948 \\
\hline C4 & -131.24 & -133.594 & -128.886 & -140.655 \\
\hline C5 & -114.727 & -108.463 & -109.222 & -93.505 \\
\hline C6 & -115.52 & -114.734 & -115.52 & -100.589 \\
\hline C7 & -95.874 & -99.017 & -99.017 & -106.09 \\
\hline C8 & -83.3 & -89.587 & -87.229 & -105.304 \\
\hline C9 & -71.512 & -76.227 & -75.441 & -89.586 \\
\hline C10 & -90.373 & -96.66 & -96.66 & -113.948 \\
\hline
\end{tabular}

The above Table-14 gives the column steel for ground storey columns w.r.t fixed base building.

Table -15: Beam Top Steel in $\mathrm{Kg}$

\begin{tabular}{|l|l|l|l|l|l|}
\hline \multicolumn{6}{|l|}{ Beam Top Steel In Kg } \\
\hline $\begin{array}{l}\text { BEAM } \\
\text { NO }\end{array}$ & FBB & TYPE I & TYPE II & TYPE III & TYPE IV \\
\hline B1 & 18.103 & 10.226 & 11.209 & 10.288 & 14.468 \\
\hline B2 & 19.551 & 10.236 & 11.091 & 10.223 & 13.488 \\
\hline B3 & 15.128 & 7.969 & 7.969 & 7.968 & 8.179 \\
\hline B4 & 14.906 & 7.052 & 7.414 & 7.128 & 8.868 \\
\hline B5 & 17.026 & 8.908 & 8.969 & 8.938 & 9.081 \\
\hline B6 & 17.144 & 9.089 & 9.466 & 9.169 & 10.771 \\
\hline B7 & 17.764 & 10.159 & 9.386 & 9.486 & 12.068 \\
\hline B8 & 17.327 & 8.291 & 9.259 & 8.281 & 11.978 \\
\hline B9 & 14.85 & 7.061 & 7.423 & 7.142 & 8.786 \\
\hline B10 & 17.899 & 10.948 & 12.296 & 10.968 & 10.612 \\
\hline
\end{tabular}

The beam top steel for ground storey beams are compared for all 4 cases in the above Table -15

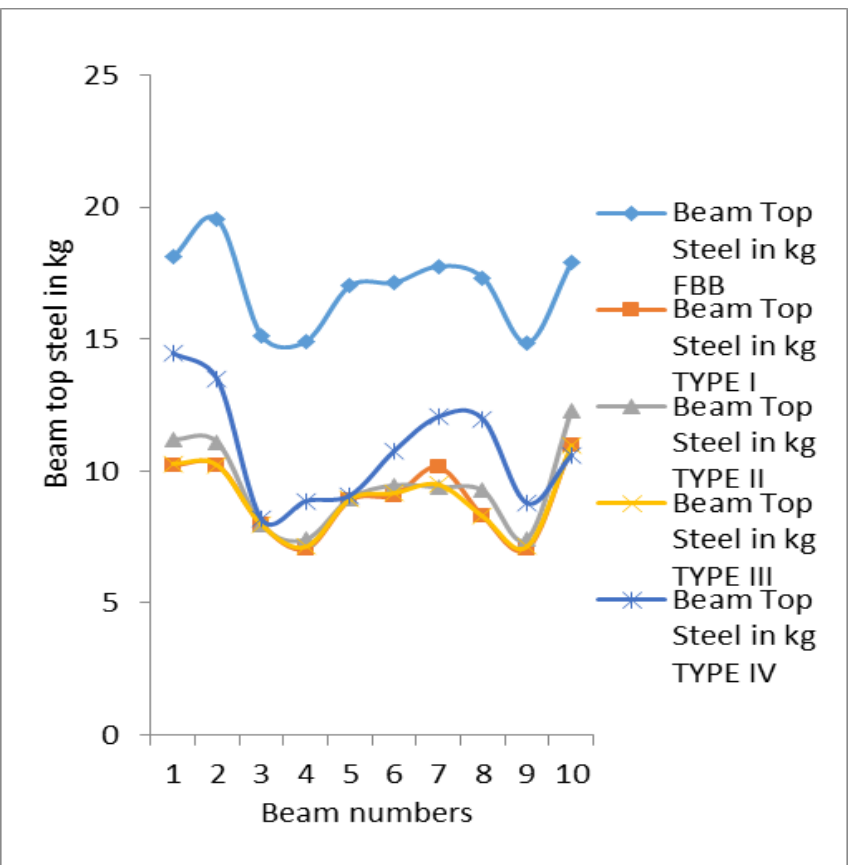

Chart -5: Beam Top Steel Values.

The chart shows the beam top steel. From the chart, we can see that the beam top steel for base isolated building is reduced when compared to fixed base building. The steel values for TYPE IV isolator is more, when compared to other isolators.

Table -16: Beam Top Steel in $\mathrm{Kg}$

\begin{tabular}{|c|c|c|c|c|}
\hline \multicolumn{5}{|c|}{ Beam Top Steel In Kg w.r.t fixed base building } \\
\hline BEAM NO. & TYPE I & TYPE II & TYPE III & TYPE IV \\
\hline B1 & -7.877 & -6.894 & -7.815 & -3.635 \\
\hline $\mathrm{B} 2$ & -9.315 & -8.46 & -9.328 & -6.063 \\
\hline B3 & -7.159 & -7.159 & -7.16 & -6.949 \\
\hline B4 & -7.854 & -7.492 & -7.778 & -6.038 \\
\hline B5 & -8.118 & -8.057 & -8.088 & -7.945 \\
\hline B6 & -8.055 & -7.678 & -7.975 & -6.373 \\
\hline B7 & -7.605 & -8.378 & -8.278 & -5.696 \\
\hline B8 & -9.036 & -8.068 & -9.046 & -5.349 \\
\hline B9 & -7.789 & -7.427 & -7.708 & -6.064 \\
\hline $\mathrm{B} 10$ & -6.951 & -5.603 & -6.931 & -7.287 \\
\hline
\end{tabular}


Table -17: Beam top Steel in $\mathrm{Kg} / \mathrm{m}^{3}$

\begin{tabular}{|l|l|l|l|l|l|}
\hline \multicolumn{5}{|l|}{ Beam Top Steel In Kg/M } \\
\hline $\begin{array}{l}\text { BEAM } \\
\text { NO }\end{array}$ & FBB & $\begin{array}{l}\text { TYPE } \\
\text { I }\end{array}$ & $\begin{array}{l}\text { TYPE } \\
\text { II }\end{array}$ & $\begin{array}{l}\text { TYPE } \\
\text { III }\end{array}$ & $\begin{array}{l}\text { TYPE } \\
\text { IV }\end{array}$ \\
\hline B1 & 47.992 & 27.112 & 29.713 & 27.269 & 38.361 \\
\hline B2 & 51.831 & 27.139 & 29.402 & 27.092 & 35.784 \\
\hline B3 & 43.272 & 22.798 & 22.798 & 22.789 & 23.386 \\
\hline B4 & 52.903 & 24.982 & 26.321 & 25.312 & 31.483 \\
\hline B5 & 55.66 & 29.121 & 29.329 & 29.213 & 29.691 \\
\hline B6 & 56.046 & 29.712 & 30.958 & 29.956 & 35.182 \\
\hline B7 & 56.583 & 32.358 & 29.895 & 30.218 & 38.424 \\
\hline B8 & 49.563 & 23.723 & 26.481 & 23.668 & 34.267 \\
\hline B9 & 52.707 & 25.103 & 26.329 & 25.331 & 31.236 \\
\hline B10 & 47.452 & 29.024 & 32.596 & 29.074 & 28.139 \\
\hline
\end{tabular}

Table -18: Beam top Steel $\mathrm{In} \mathrm{Kg} / \mathrm{m}^{3}$ w.r.t FBB

\begin{tabular}{|c|c|c|c|c|}
\hline \multicolumn{5}{|c|}{ Beam Top Steel In $\mathrm{Kg} / \mathrm{M}^{3}$ w.r.t FBB } \\
\hline $\begin{array}{l}\text { BEAM } \\
\text { NO }\end{array}$ & $\begin{array}{l}\text { TYPE } \\
\text { I }\end{array}$ & $\begin{array}{l}\text { TYPE } \\
\text { II }\end{array}$ & $\begin{array}{l}\text { TYPE } \\
\text { III }\end{array}$ & $\begin{array}{l}\text { TYPE } \\
\text { IV }\end{array}$ \\
\hline B1 & -20.88 & -18.279 & -20.723 & -9.631 \\
\hline B2 & -24.692 & -22.429 & -24.739 & -16.047 \\
\hline B3 & -20.474 & -20.474 & -20.483 & -19.886 \\
\hline B4 & -27.921 & -26.582 & -27.591 & -21.42 \\
\hline B5 & -26.539 & -26.331 & -26.447 & -25.969 \\
\hline B6 & -26.334 & -25.088 & -26.09 & -20.864 \\
\hline B7 & -24.225 & -26.688 & -26.365 & -18.159 \\
\hline B8 & -25.84 & -23.082 & -25.895 & -15.296 \\
\hline B9 & -27.604 & -26.378 & -27.376 & -21.471 \\
\hline B10 & -18.428 & -14.856 & -18.378 & -19.313 \\
\hline
\end{tabular}

Table -19: Beam Bottom Steel in $\mathrm{Kg}$

\begin{tabular}{|l|l|l|l|l|l|}
\hline \multicolumn{5}{|l|}{ Beam Bottom Steel In Kg } \\
\hline $\begin{array}{l}\text { BEAM } \\
\text { NO }\end{array}$ & FBB & $\begin{array}{l}\text { TYPE } \\
\text { I }\end{array}$ & $\begin{array}{l}\text { TYPE } \\
\text { II }\end{array}$ & $\begin{array}{l}\text { TYPE } \\
\text { III }\end{array}$ & $\begin{array}{l}\text { TYPE } \\
\text { IV }\end{array}$ \\
\hline B1 & 9.051 & 5.113 & 5.598 & 5.146 & 7.251 \\
\hline B2 & 9.775 & 5.118 & 5.545 & 5.112 & 6.736 \\
\hline B3 & 7.564 & 3.985 & 3.895 & 3.894 & 4.078 \\
\hline B4 & 7.453 & 3.526 & 3.698 & 3.568 & 4.425 \\
\hline B5 & 8.513 & 4.454 & 4.485 & 4.465 & 4.536 \\
\hline B6 & 8.572 & 4.544 & 4.726 & 4.586 & 5.378 \\
\hline B7 & 8.882 & 5.08 & 4.694 & 4.738 & 6.028 \\
\hline B8 & 8.664 & 4.145 & 4.627 & 4.137 & 5.978 \\
\hline B9 & 7.425 & 3.531 & 3.665 & 3.566 & 4.386 \\
\hline B10 & 8.95 & 5.474 & 6.091 & 5.487 & 5.421 \\
\hline
\end{tabular}

The beam bottom steel for ground storey beams are taken from SAP analysis results for each case are compared for all 4 cases in the above Table 19

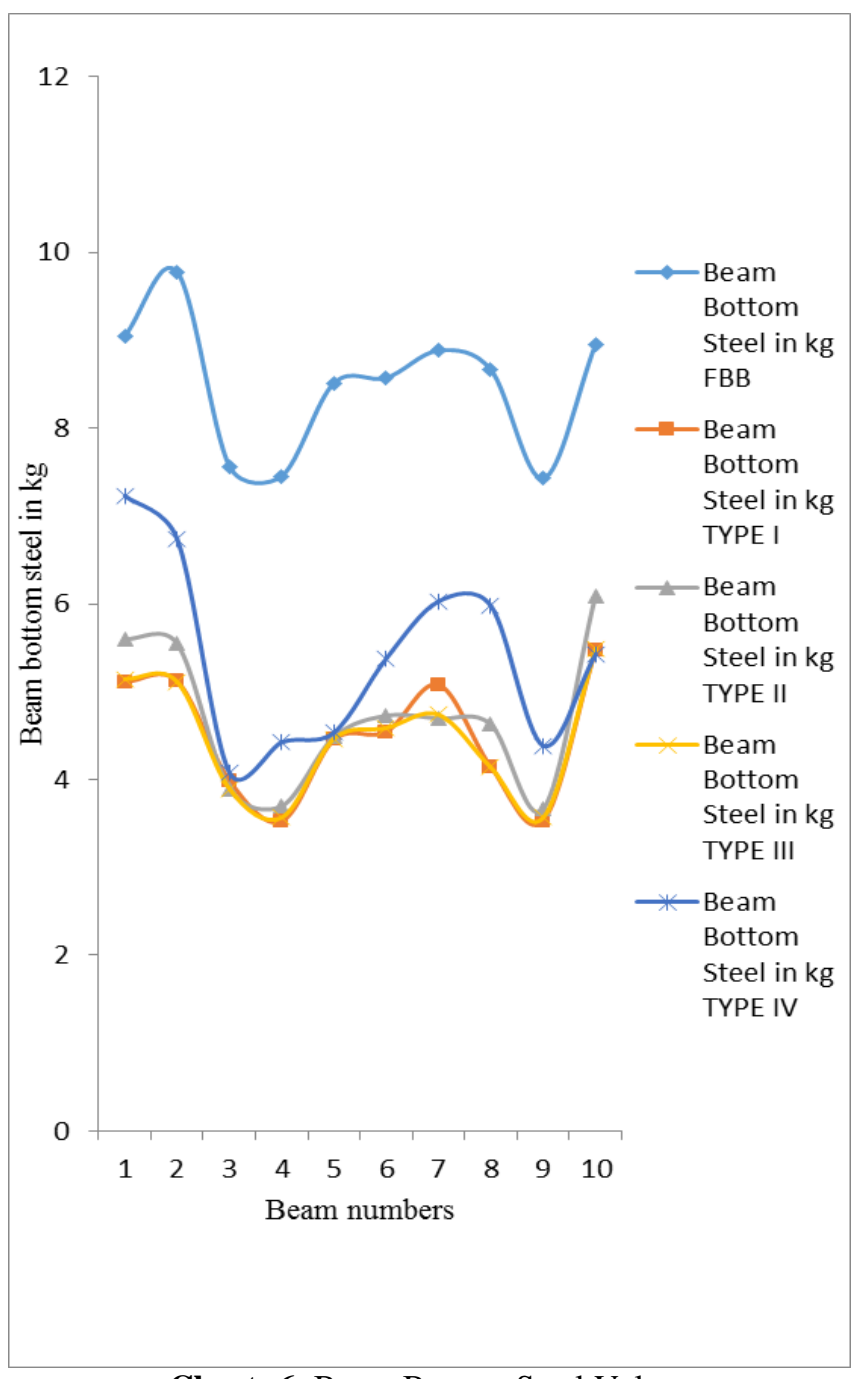

Chart -6: Beam Bottom Steel Values 
The chart -6 shows the beam bottom steel. From the chart we can see that the beam bottom steel for base isolated building is reduced when compared to fixed base building. The steel values for TYPE IV isolator is more, when compared to other isolators.

Table -20: Beam Bottom Steel in $\mathrm{Kg}$

\begin{tabular}{|l|l|l|l|l|}
\hline \multicolumn{5}{|c|}{ Beam Bottom Steel In Kg w.r.t FBB } \\
\hline $\begin{array}{l}\text { BEAM } \\
\text { NO }\end{array}$ & TYPE I & TYPE II & TYPE III & TYPE IV \\
\hline B1 & -3.938 & -3.453 & -3.905 & -1.823 \\
\hline B2 & -4.657 & -4.23 & -4.663 & -3.039 \\
\hline B3 & -3.579 & -3.669 & -3.67 & -3.486 \\
\hline B4 & -3.927 & -3.755 & -3.885 & -3.028 \\
\hline B5 & -4.059 & -4.028 & -4.048 & -3.977 \\
\hline B6 & -4.028 & -3.846 & -3.986 & -3.194 \\
\hline B7 & -3.802 & -4.188 & -4.144 & -2.854 \\
\hline B8 & -4.519 & -4.037 & -4.527 & -2.686 \\
\hline B9 & -3.894 & -3.76 & -3.859 & -3.039 \\
\hline B10 & -3.476 & -2.859 & -3.463 & -3.529 \\
\hline
\end{tabular}

Table -21: Beam Bottom Steel in $\mathrm{Kg} / \mathrm{m}^{3}$

\begin{tabular}{|l|l|l|l|l|l|}
\hline \multicolumn{7}{|c|}{ Beam Bottom Steel In $/ \mathrm{m} 3$} \\
\hline $\begin{array}{l}\text { BEAM } \\
\text { NO }\end{array}$ & FBB & $\begin{array}{l}\text { TYPE } \\
\text { I }\end{array}$ & $\begin{array}{l}\text { TYPE } \\
\text { II }\end{array}$ & $\begin{array}{l}\text { TYPE } \\
\text { III }\end{array}$ & $\begin{array}{l}\text { TYPE } \\
\text { IV }\end{array}$ \\
\hline B1 & 23.996 & 13.555 & 14.861 & 13.642 & 19.183 \\
\hline B2 & 25.916 & 13.568 & 14.701 & 13.542 & 17.886 \\
\hline B3 & 21.636 & 11.398 & 11.397 & 11.396 & 11.687 \\
\hline B4 & 26.452 & 12.515 & 13.158 & 12.652 & 15.731 \\
\hline B5 & 27.831 & 14.561 & 14.668 & 14.611 & 14.843 \\
\hline B6 & 28.023 & 14.856 & 15.478 & 14.978 & 17.586 \\
\hline B7 & 28.291 & 16.179 & 14.013 & 15.112 & 19.212 \\
\hline B8 & 24.781 & 11.857 & 13.241 & 11.832 & 17.129 \\
\hline B9 & 26.353 & 12.537 & 13.169 & 12.671 & 15.613 \\
\hline B10 & 23.726 & 14.512 & 16.301 & 14.532 & 14.372 \\
\hline
\end{tabular}

\section{CONCLUSIONS}

The highly functional structures like Bridges, power plants, buildings can be protected from a technology called Base isolation. The main purpose of this work is to study the dynamic response of structure when subjected to different isolators and cost of base isolated structure when subjected to strong seismic forces in order to pursue an optimal design of the system. For the analysis of different isolation systems, three-dimensional RC building, with base isolation devices under each column have been investigated.

According to the analytical study following conclusions were drawn:

1. The Base isolation substantially increases the time period of the building \& hence correspondingly reduces the base shear. As observed in Table $-2 \&$ Table -3 the time period is being increased upto 9.4 times \& base shear is reduced upto $1 / 5^{\text {th }}$ of that of fixed one.

2. By considering the bending moment values in Table -5 it is clearly observed that for isolated cases the maximum bending moment is reduced as that of fixed case. So considering bending moment also, the high damping rubber isolated frame is performing better as compared to the other isolator stiffness.

3. The shear force values in Table -7 shows that the ground storey columns are reduced for isolated frame when compared fixed base building.

4. From the analytical results, it is observed that how effective seismic isolation works by considering various aspects such as: base shear, maximum bending moments, \& column shears, etc. Analysis results of the study suggest that high damping rubber isolators are better option as compared to other isolators.

\section{REFERENCES}

[1]. Gomase O.P, Bakre S.V, "Performance of NonLinear Elastomeric Base-Isolated building structure", International journal of civil and structural engineering volume 2, no 1, 2011.

[2]. Khante.S.N , Lavkesh R.Wankhade, "Study of seismic response of symmetric and asymmetric base isolated building with mass asymmetry in plan", International journal of civil and structural engineering volume $\mathrm{x}$, no $\mathrm{x}, 2010$.

[3]. Gokhan Ozdemir n,1,MichaelC.Constantinou," Evaluation of equivalent lateral force procedure in estimating seismic isolator displacements". Elsevier, Soil Dynamics and Earthquake Engineering 30 (2010) 1036-1042.

[4]. Vojko Kilar, David Koren," Seismic behavior of asymmetric base isolated structures with various distributions of isolators". Elsevier, Engineering Structures 31 (2009) 910_921.

[5]. Pourzeynali.s, Zarif.M, " Multi-objective optimization of seismically isolated high-rise building structures using genetic algorithms". Elsevier, Journal of Sound and Vibration 311 (2008) 1141-1160.

[6]. Ozpalanlar.G, "Earthquake response analysis of midstory buildings isolated with various seismic isolation techniques", The $14^{\text {th }}$ World Conference on Earthquake Engineering October 12-17, 2008, Beijing, China.

[7]. Panayiotis C. Polycarpou, Petros Komodromo," Earthquake-induced poundings of a seismically isolated building with adjacent structures". Elsevier, 
Engineering Structures 32 (2010) 1937_1951.

[8]. Juan C. de la Llera "Experimental behavior and design of a new kinematic isolator", Engineering Structures 32 (2010) 508_522.

[9]. Arturo Tena-Colunga, Christian Zambrana-Rojas," Dynamic torsional amplifications of base isolated structures with an eccentric isolation system". Elsevier, Engineering Structures 28 (2006) 72-83.

[10]. Kalantari S.M. "Investigation of base-isolator type selection on seismic behavior of structures including story drifts and plastic hinge formation", The $14^{\text {th }}$ World Conference on Earthquake Engineering October 12-17, 2008, Beijing, China.

[11]. C.P. Providakis," Effect of LRB isolators and supplemental viscous dampers on seismic isolated buildings under near-fault excitations". Elsevier, Engineering Structures 30 (2008) 1187-1198.

[12]. Agarwal V.K. "Earthquake induced pounding in friction varying base isolated buildings", Engineering Structures 29 (2007) 2825-2832. 